\title{
Food Safety Informatics: A Public Health Imperative
}

\author{
Cynthia A. Tucker, PhD., MBA., RD., LDN., ${ }^{1}$ Stephanie N. Larkin, B.S., MBAc, ${ }^{2}$ \\ Timothy A. Akers, M.S., Ph.D. ${ }^{3}$
}

${ }^{1}$ Nutritional Science Program, School of Community Health and Policy, Morgan State University

${ }^{2}$ School of Business, Morgan State University

${ }^{3}$ School of Computer, Mathematical and Natural Sciences, and Center for Health

Informatics, Planning and Policy (CHIPP), Morgan State University

\section{Abstract}

To date, little has been written about the implementation of utilizing food safety informatics as a technological tool to protect consumers, in real-time, against foodborne illnesses. Food safety outbreaks have become a major public health problem, causing an estimated 48 million illnesses, 128,000 hospitalizations, and 3,000 deaths in the U.S. each year. Yet, government inspectors/regulators that monitor foodservice operations struggle with how to collect, organize, and analyze data; implement, monitor, and enforce safe food systems. Currently, standardized technologies have not been implemented to efficiently establish "near-in-time" or "just-in-time" electronic awareness to enhance early detection of public health threats regarding food safety. To address the potential impact of collection, organization and analyses of data in a foodservice operation, a wireless food safety informatics (FSI) tool was pilot tested at a university student foodservice center. The technological platform in this test collected data every six minutes over a 24 hour period, across two primary domains: time and temperatures within freezers, walk-in refrigerators and dry storage areas. The results of this pilot study briefly illustrated how technology can assist in food safety surveillance and monitoring by efficiently detecting food safety abnormalities related to time and temperatures so that efficient and proper response in "real time" can be addressed to prevent potential foodborne illnesses.

Key words: foodborne illness, surveillance, technology 


\section{Introduction}

Foodborne illness sees no boundaries. The world has witnessed, first hand, the devastating public health impact of foodborne illnesses that have occurred in Europe. The foods that were impacted through this contamination spread quickly and with fatal outcomes affecting nearly 4,023 who became ill, 48 deaths and 885 diagnosed with Hemolytic Uremic Syndrome [4]. Results from this outbreak has made it is clear that food safety has advanced to become a world public health problem.

Food safety in the United States has been a major concern for decades. Consequently, congress passed the "FDA Food Safety Modernization Act of 2010" and it was signed into law by President Obama on January 4, 2011. The passing of this Act is the first major overhaul of the nation's food safety systems in over 70 years. This legislation represents a major reform of the original food safety requirements of the Federal Food, Drug, and Cosmetic Act (FD\&C Act) of 1938, by expanding the powers of the Food and Drug Administration (FDA) with respect to food safety and food security.

Every year, the U.S. Centers for Disease Control and Prevention (CDC) estimates that tens of millions of Americans fall ill, hundreds of thousands are hospitalized, and thousands die from foodborne illnesses [10]. According to the CDC, unsafe foods cause an estimated 48 million illnesses, 128,000 hospitalizations, and 3,000 deaths each year in the United States [3]. The financial impact of foodborne illness in the United States is estimated at a staggering \$152 billion per year due to sickness, lost of productivity, loss of human life and destruction to entire industries and regional economies [11].

For example, food safety has been at the forefront of media publicity in recent years. Many high-profile outbreaks of contaminated food include peanuts and peanut-related products, tomatoes, peppers, spinach, and other foods $[15,16,17]$. In fact, in the summer and fall of 2010 , over 32 million cartons of eggs were recalled due to salmonella contamination [5-6]. As a result of these incidences, food safety has become a growing concern for consumers, the food industry and government.

In theory, to address the potential impact of further foodborne outbreaks, the federal food regulators are aiming to change the focus of food safety and contamination from a reactive approach to a more rapid response, prevention, and detection strategy. The impact of the recent legislation transcends policy, public health, industry, and technology. It is anticipated that policy-makers, public health analysts, and technologists will soon be left to consider how best to collect, organize, implement, monitor, enforce and analyze these new legislative mandates. However, to date, there does not exist a working definition, description, or processes regarding "Food Safety Informatics" as a conceptual and practical framework or paradigm. Creating an appreciation for Food Safety Informatics can help to develop and shift our analytical ability to better understand how to integrate epidemiologic investigations with foodborne outbreak and contamination analyses [18]. Our working definition of "Food Safety Informatics" blends human factors with technology through the use of standardization of data elements when collecting data electronically for risk tracking, surveillance, and analysis. 
While these new legislative and technological changes are essential for national regulatory food safety monitoring of tainted foods from the producers, this only addresses half of the risk. There still exist a critical public health and food safety gap that is often overlooked and goes unanswered between the producers of food products and the consumers: the eating establishments where the public consumes over 70 billion meals annually at schools, restaurants, retirement homes, universities, businesses, government cafeterias, and other public eating venues [5-6]. It is at the local, community level where food safety is the greatest public health imperative.

Foodborne incidences have followed a "reactive state of response" by looking back-in-time to determine what could have been the cause of an outbreak. Though what have been needed are "real-time" alerts and responsive public health and food safety systems at the local level. Currently, governmental inspectors, primarily through local health departments, depend primarily on paper-based documentation provided by businesses or food preparers to verify that the foods to be served are free of contamination. Traditionally, recurring violations have been handled through re-inspections or in some cases, fines, suspension of permits, or closures have been enforced.

However, there are far too many foodservice businesses, such as restaurants or cafeterias that do not implement proactive systems of controls to prevent violations from reoccurring, thereby, consistently repeating food safety hazards. This is critical because the various methods employed across the food industry have been unsustainable and inconsistent when implementing preventive controls (including critical control points), active monitoring, verification, corrective actions, and accurate and reliable recordkeeping [14].

To date, little has been written about the implementation of utilizing food safety informatics as a technological tool to protect consumers, in real-time, against foodborne illnesses. Widespread standardized technologies have not been implemented to efficiently establish near-in-time, or "just-in-time," electronic situational awareness to enhance early detection of, rapid response to, and management of public health threats toward food safety [9]. Moreover, the lack of a broad public health preventative strategy has resulted in the limited ability or failure to collect, analyze and disseminate systematic information related to food safety issues across organizational and jurisdictional boundaries.

\section{Institutional Risk: A public health imperative}

School cafeterias, restaurants and food service facilities serve millions of meals every day, and usually these meals are safe to consume. However, when food safety problems occur, they are especially dangerous to children, $[1,2,7,8,10,12,13,15]$ and people with compromised immune systems due to disease (i.e., HIV/AIDS, cancer, cystic fibrosis, etc.), [8] or patients receiving pharmacologic therapy (i.e., chemotherapy, radiation), [12]. Everyday consumers attending public eateries are subjected to the risk of foodborne contaminants, ranging from spoiled food products to the negligent washing of hands.

Through these experiences public health is confronted with a myriad of challenges, a complex taxonomy of risks that can go unexamined without standardized data collection systems. The 
most significant challenge to establish a public health imperative has been educating policy makers and federal, state, and local regulators about the hazards of contaminated foods that are consumed by people in both public and private foodservice facilities. In contrast, if mandated, industry adapts quickly when evidence-based policy helps to guide industry down a path of efficiency. Given that significant food safety gaps exist within the current public health monitoring systems, government regulators struggle to monitor the most fundamental aspects of food supply systems, maintenance, and operations.

Response times in tracing tainted food or non-sanitized environments place the public in serious risk of foodborne illness outbreaks. This has been an ongoing challenge facing the food industry, public health and regulatory agencies. The complexity of these issues increase exponentially when considering the various dimensions to the problem, along with the lack of more sophisticated and state-of-the-art technologies that have the capabilities and capacity to captures real-time food safety data, leading to a rapid and timely interdiction, if a problem is detected.

To better understand these shortcomings, a systematic review of the literature was conducted. However, what was achieve was an awareness that significant gaps and deficiencies in the context of public health and food safety technology and their relationship to new advances in monitoring and reporting exist throughout the literature. Very little has been written on this interrelationship.

\section{Methods}

To address the research deficit and begin to build a body of public health knowledge, the authors of this paper propose to introduce new food safety surveillance and monitoring technology through a pilot study. In the initial phase of identifying a food safety informatics tool, we worked closely with the technology industry to identify a leading food safety data acquisition technology. To identify a particular type of technology, we presented a particular set of detailed criteria to the technology industry. The technology had to be automated, able to automatically collect and transmit its data wirelessly; acquire real-time temperature data, both through automation and manually; and obtain standardized facility's and maintenance checklist data at regular time intervals. Lastly, the data output had to be in graphic form with real-time notification capabilities. The food safety data acquisition technology identified was termed Touchblock $^{T M}$ (Figure 1).

\section{Setting}

In the summer of 2010 , to test the food safety informatics tool, the authors of this study developed research collaborations with an industry leader in advanced food safety technology, and with a highly respected national food service caterer who provided services to a Historically Black College and University (HBCU), located in the eastern United States. 


\section{Study Design}

This collaboration included a pilot study test of monitoring time and temperatures utilizing the food safety informatics tool Touchblock ${ }^{T M}$. During this pilot, no human subjects were included in this study nor were any existing operations or technologies changed or altered. There was no outside interference whatsoever. Over the course of a 24-hour period of time, automated and remote, wireless monitoring and transmitting technology were installed in industrial grade, walkin refrigerators, freezers, and dry storage. The sensor monitors were designed to collect data every 6 minutes throughout the 24 hour period; however to see the data easier, and clearer, 2hour time intervals are reported over the course of 12 hours. The wireless remote sensors were strategically place in all three of the test locations (freezer, walk-in refrigerator and dry storage).

Where the food was stored served as our unit of analysis and sample. The food monitoring technology chosen, referred to as Touchblock ${ }^{T M}$ was designed to transmit real-time and temperature data every 6-minutes. The Touchblock ${ }^{T M}$ technological platform in this beta-test collected data across three primary domains: 1) time, 2) temperature, and 3) quality control checklists. However, for purposes of this study, only time and temperature data are reported.

\section{Instrument}

Touchblock ${ }^{T M}$ was the instrument chosen for this study because it met select criteria for inclusion (see Figure 1). The researchers of this study developed particular criteria that included technology with the capability of transmitting real-time temperature and time data. The technology had to be automated, able to automatically collect and transmit its data wirelessly; acquire real-time temperature data, both through automation and manually; and obtain standardized facility's and maintenance checklist data at regular time intervals. The data output had to be in graphic form with real-time notification capabilities and assessable for surveillance 24 hours a day. Lastly, overall data integrity should allow users the ability to conduct Hazard Analysis Critical Control Point type analysis whereby, root cause analysis and corrective action plans can be developed using quantifiable data that is built upon evidenced-based scientific theory and outcomes.

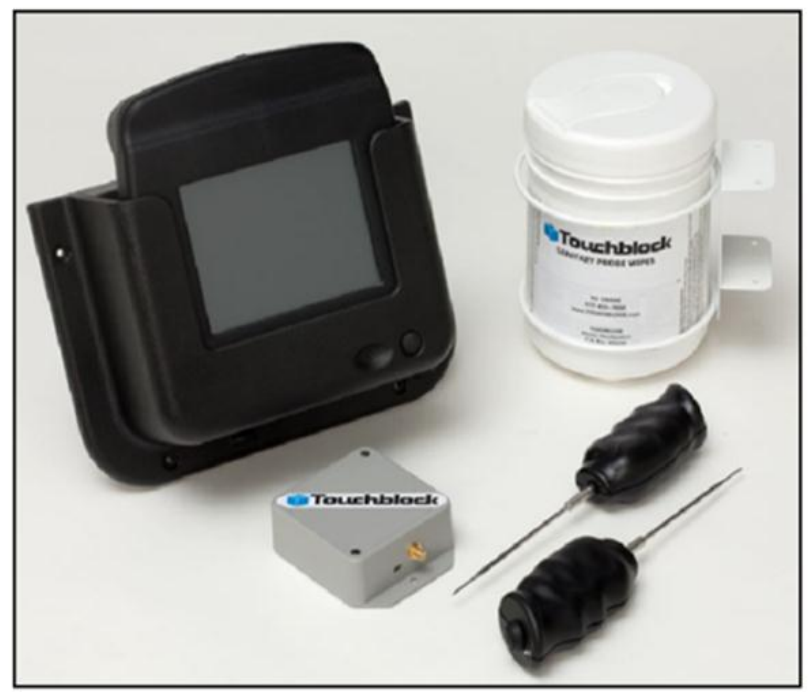

Figure 1. Touchblock Handheld Food Monitoring Data, Remote, and Manual Sensors 


\section{Results}

The data collected over the 24 hour period illustrates how reporting food safety data can serve as a real-time food safety monitoring surveillance system for foodservice establishments and public health inspectors. Figure 2a represents the surveillance of freezer temperature changes over a 12 hour period. The freezer normal operating range was established from minus 15 to 15 degree Fahrenheit. As illustrated in Figure 2a, this particular freezer experienced peaks far exceeding its acceptable range, to a peak of approximately 26 degrees Fahrenheit. We further investigated this time frame to determine if a delivery had been received and consequently was being stored or was it a physical inventory that could explain the increase in temperature. Neither was the case.

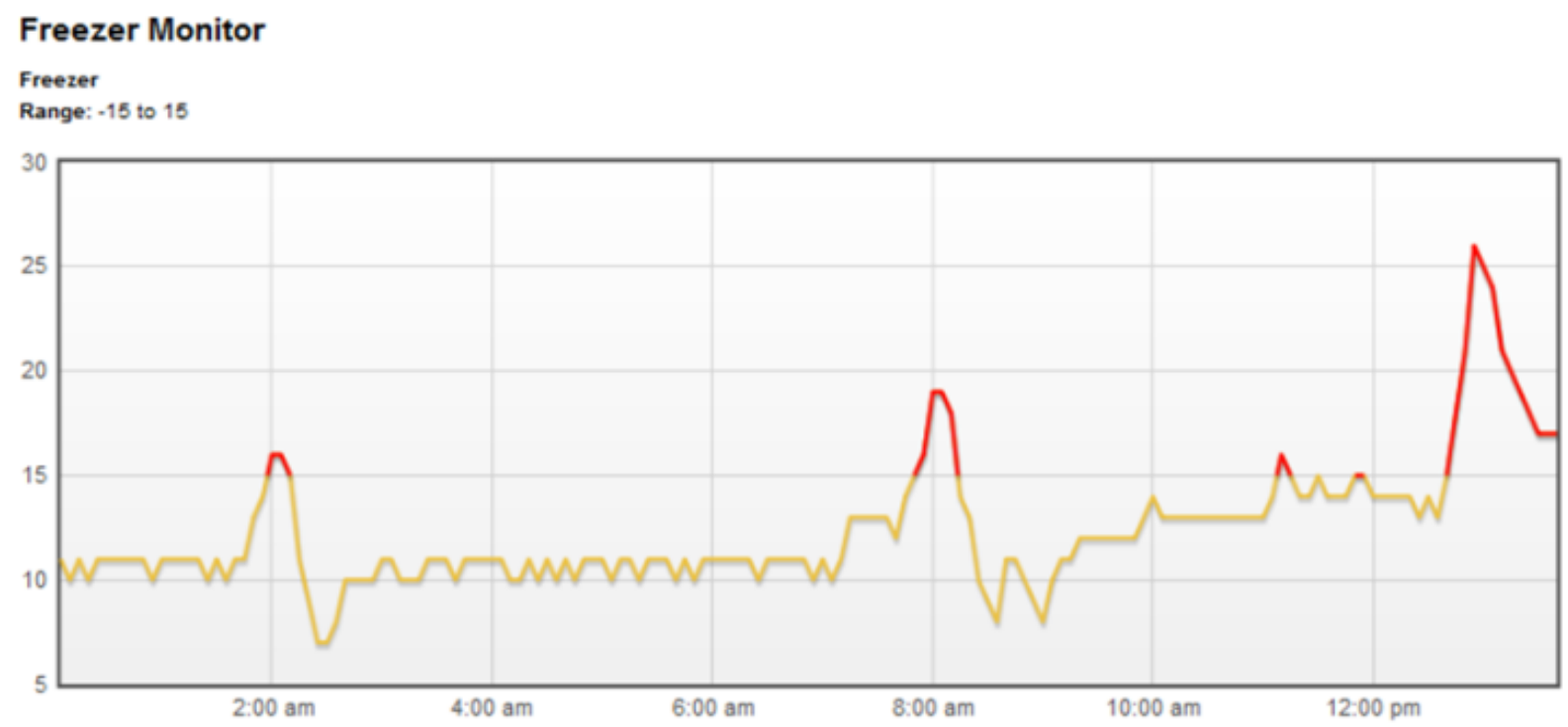

Figure 2a-Automatic Freezer Monitor 1

Color code: Yellow $=$ normal range Red $=$ above normal range

Figure $2 \mathbf{b}$ show that the acceptable temperature ranges for refrigerator monitor 2 for the foodservice business was between 35 and 41 degrees. However, it is clear that Figure $\mathbf{2 b}$ shows that there were significant time intervals in which the temperatures far exceeded the acceptable range for prolonged periods of time, peaking at 48 degrees. Based on the time, it is clear that these peak changes were most likely due to foodservice workers entering and leaving the coolers. 


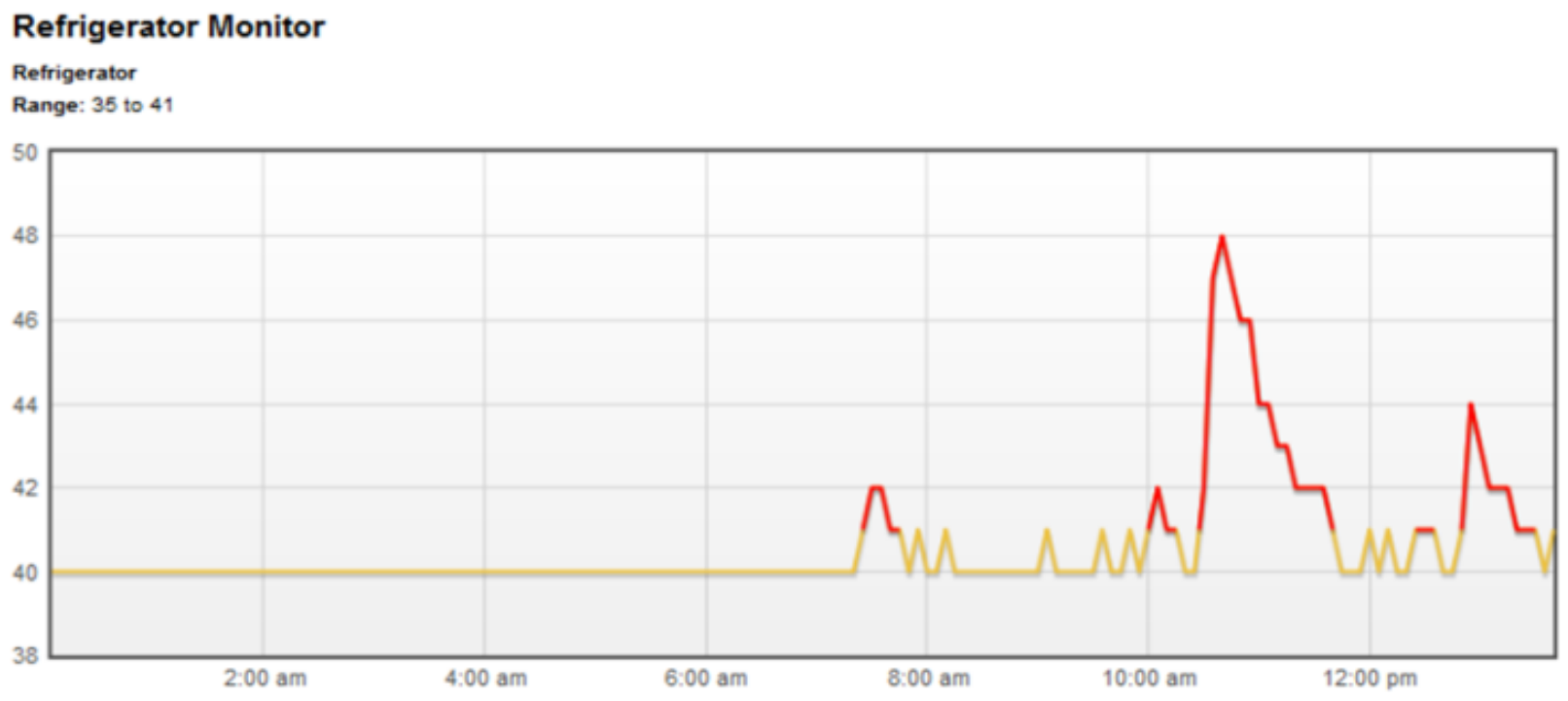

Figure 2b--Automatic Refrigerator Monitor 2.

Color code: Yellow $=$ normal range, Red $=$ above normal range

Normal range for dry storage should have been from 70 to 75 degrees. However, Figure 2c shows over the course of the night and throughout the day, the dry storage monitoring sensors indicate that the temperatures exhibit a peak of 100 degrees and never dropping below 84 degrees Fahrenheit, which far exceeded the standard temperature range for the industry.

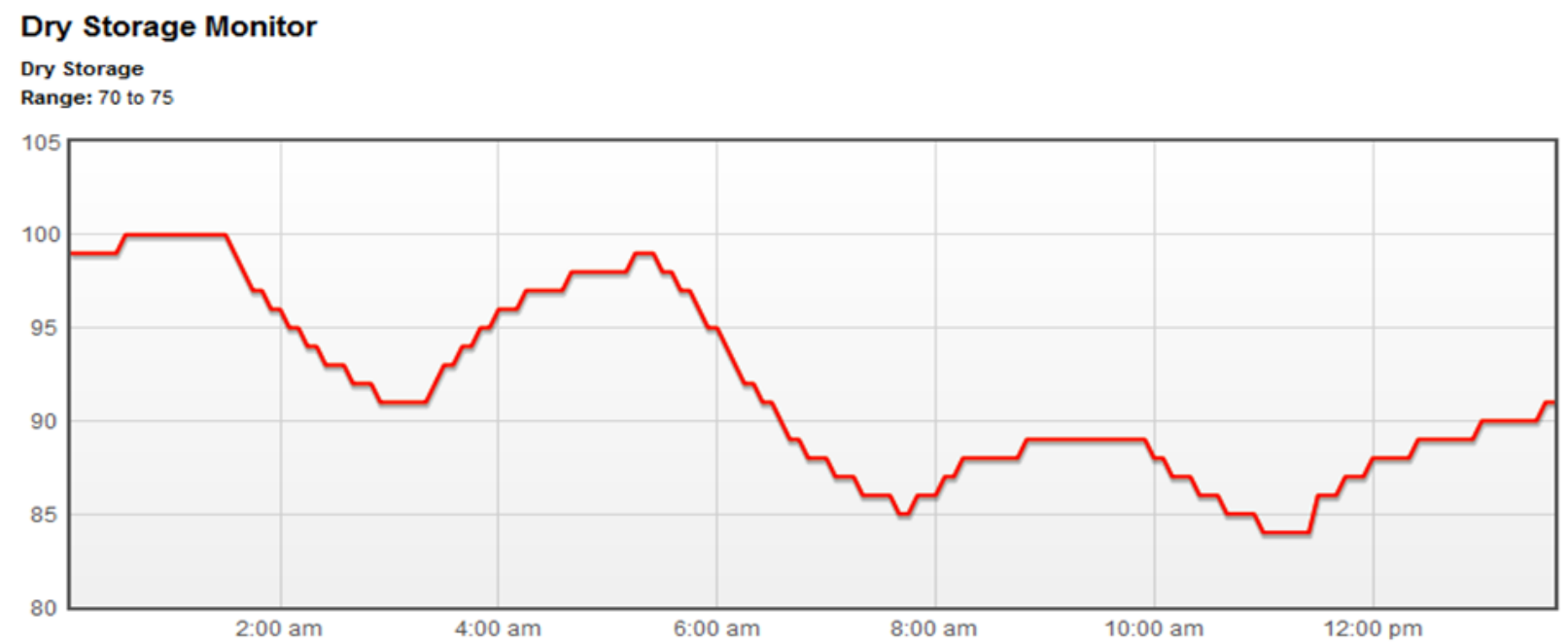

Figure 2c-Dry Storage Room Monitor 3.

Color code: Red $=$ above normal range

In summary, the results of the above 24 hour pilot study briefly illustrates how technology can assist in food safety surveillance and monitoring by efficiently detecting food safety abnormalities related to time and temperatures. Through the use of Touchblock, temperatures outside the accepted range can be quantified, over time, so that efficient and proper response in 
"real time" can be addressed to prevent microbial growth, and/or spoilage of products resulting in loss of product inventory and potential foodborne illnesses.

\section{Discussion}

Since foodborne illness sees no boundaries, it is imperative that those who track it embrace the overall technological concept of food safety informatics surveillance. Based on our analysis of this Food Safety Informatics pilot study, the automated food safety data acquisition technology served to validate the food safety informatics tool. With data being collected, in real-time, through automated wireless sensors, the ability of the industry and food protection regulators to monitor the potential impact is significantly enhanced.

Collecting accurate time and temperature data, to include facility maintenance data of their normal business checklist, far exceeds paper-based data reporting. Current normal procedures utilizing paper-based data systems are highly suspect, subject to manipulation, and unreliable. However, the use of date and time-stamped temperature monitoring technology can serve to more readily protect the public's health against foodborne contaminants due to spoiled foods or other risk issues. In addition, using food safety informatics enables a foodservice operation to download the collected surveillance data into a centralize repository where data can be stored for review, analyzed, interpreted and utilized for reporting purposes. This innovative method of collecting data substantially increases the organization's creditability with government food safety inspectors thereby, exemplifying their committed efforts to protect the consumer from foodborne illnesses.

Food Safety Informatics surveillance systems will soon be found essential in dealing with the myriad of challenges that the foodservice industry face. Users of the current "paper" $\log$ recordkeeping methods will find that the food safety informatics system will be more straightforward and consistent with a high degree of integrity, accuracy, and reliability. Currently, foodservice operations are required to keep a "paper" log of daily time and temperatures. This log is done by hand using a handheld thermometer probe or reading the temperature gauge on freezers or refrigerators. In many instances the temperatures recorded are fraudulently or artificially made up to ensure health code compliance. Food Safety Informatics will minimize the variables of human error in temperature gathering, recording, and transposing data from one source to another and most importantly, allow for faster response time to potential food safety issues or events.

An important key advantage of utilizing a food safety informatics surveillance system is that time and temperature data are continuously collected 24 hours a day, 7 days a week. At any given time, a proper authorized user can access the collected surveillance data of food service cooling, freezing and refrigeration equipment to make sure proper holding temperature ranges are maintained and if for some reason they are not, efficient and proper response can be coordinated and applied.

Other uses of the food safety informatics surveillance system go beyond monitoring equipment and storage areas for food. The Touchblock ${ }^{T M}$ informatics technological tool is also designed to replace all other "paper" logs that are used for basic facility check list maintenance reports (e.g., 
time lavatory cleaned, countertops sanitized, food rotations, etc.) and virtually all other temperature logs of prepared foods that are expected to be done by hand using a hand-held thermometer probe. Figure 3 illustrates how data are collected for the purposes of food safety informatics, as opposed to the paper-based copies.
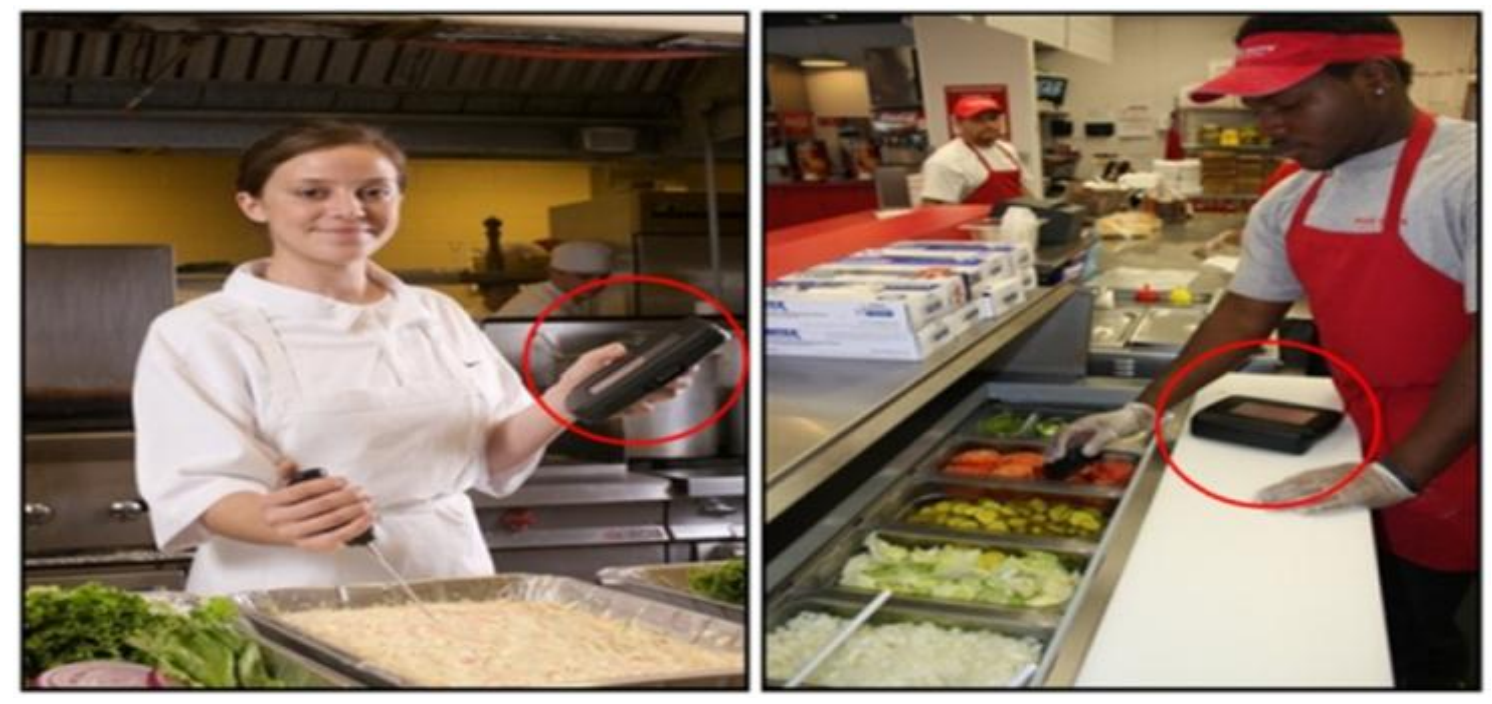

Figure 3. Automatic Handheld Probes for Temperature Collection and Transmission

Manual probes allow for verifiable temperature monitoring of prepared foods outside refrigerated areas to ensure proper holding temperatures that are maintained in accordance with health code requirements. Critical products, containing temperature sensitive and biological components (i.e., meats, poultry, seafood, cream dishes, gravies etc.) can be checked with the manual probe to ensure that proper temperatures are either maintained or achieved. The automated surveillance informatics tool will also be time stamped and recorded to guarantee data accuracy and integrity.

In summary, today's food safety technology is sorely underutilized or not used at all and must be developed and introduced into all segments of the food industry. The benefits of a food safety informatics surveillance system are the collection of automated data that can be used as a proactive agent rather than a reactive agent in the prevention of foodborne illnesses. In addition, the use of automation for data collection minimizes human reporting errors and increases the integrity of required recordkeeping. Technology cannot totally stop intentional or unintentional food related outbreaks from occurring, however technological advances can help to make the overall food system "proactive" instead of "reactive" in protecting the public in the event of an outbreak or super-spread of a foodborne illness.

\section{Future Direction: A Proposed Food Safety Informatics Model}

Based on our proof of concept, it is clear that the public's health is weighty at risk unless new models are introduced to help identify food safety anomalies in the food service industry, 
ranging from restaurants to school cafeterias to elderly dining facilities. Whatever the venue, it is clear that public health has not delved into this body of research significantly, and a gap in literature clearly exists for the field and practice of public health in this area. For example, the evolution of "health informatics" is, in many respects, not significantly different than in the area of food safety informatics. Evidence-based food safety data can serve to identify best practices in the food industry when it comes to food preparation for immediate consumption.

Figure 4 is a diagrammatical model to illustrate how a Historically Black University is developing a national model to introduce food safety informatics as a public health imperative. Figure 4 illustrates that core surveillance should, ideally, be accessible to critical federal reporting agencies when it comes to food safety.

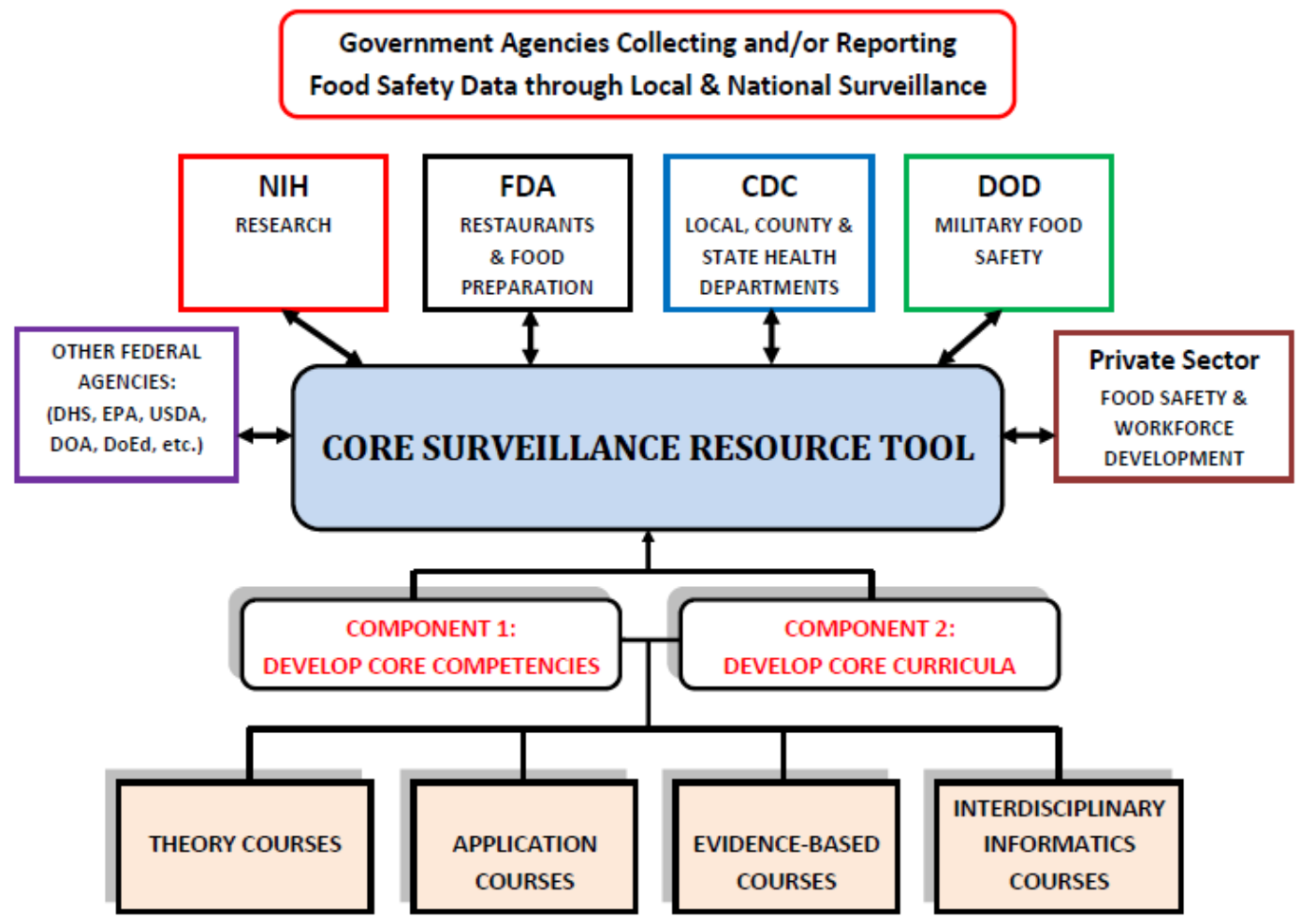

Figure 4: Food Safety Informatics Model: A New Paradigm

Through our use of the Touchblock ${ }^{T M}$ system and key informant discussions with industry, academia, and government, we have been able to develop this conceptual model of the salient issues that need to be considered when bridging this gap between food safety informatics and public health. Data collection systems, such as Touchblock, ${ }^{T M}$ have served as a single purpose centralized monitoring portal suitable for use in commercially and industrialized environments. The goal of any food safety informatics system should be to gather critical data and verify in real-time that adequate food, facility equipment and conditions are maintained consistently and not for any one snapshot period of time, as is the industry standard convention with paper-based systems that are currently in use. 
To develop this national model, two overarching thematic issues were identified as being critical to develop a national food safety informatics model: 1) develop core competencies in food safety informatics and 2) develop curricula. Figure 4 illustrates four key areas of development: theory, application, evidence-based analysis, and interdisciplinary relevance. We will summarize the model to briefly describe their role in developing a food safety informatics model.

Theory - The theory courses encompass scientific background of foodborne pathogens, importance of personal hygiene, cross contamination and other conditions that impact food safety. In addition, examining the theory of the use of technology, a critical component in training, can be used to demonstrate its efficiency in operational procedures as it pertains to food safety and the theoretical reasons and rational why technology should evolve, starting with the replacement of existing paper-based systems.

Application - Courses that relate to application are sensitive to the foodservice workforce. Training is designed and tailored to learners of different generational ages, cultural background, literacy and technological ability with respect to different occupational applications in industry.

Evidence-based Analysis - Courses related to evidence based analyses are data driven. These courses provide "real" situational awareness modules that promote effective data gathering through mathematical and epidemiological algorithms which can help in teaching and developing predictive models thereby assisting in timely responses that best meet the challenges of food safety.

Interdisciplinary Informatics Relevance - These courses incorporate multiple academic disciplines (i.e., engineering, mathematics, computer sciences, public health, biology, etc.) to collaborate in research to solve food safety issues through innovative technology and science by utilizing technological applications that capture time series data. The importance of interdisciplinary informatics input can help to enhance subject matter expertise that often goes overlooked and neglected by any one discipline, such as public health.

In summary, the theoretical model describes food safety informatics as a public health imperative. By today's public health standards, there exist significant gaps in the nation's overall ability to monitor, teach, and standardize many of the most fundamental aspects that relate to food supply systems, maintenance, and operations. The use of technology cannot totally stop food related outbreaks from happening. However, technological advances can help to make the overall system of prevention, detection, reporting, and coordinated response more efficient in the event of a small or catastrophic foodborne illness outbreak.

\section{Conclusion}

Food safety informatics is the first line of defense for eateries to prevent foodborne outbreaks. Federal legislators and regulators have an obligation to protect consumers against unanticipated foodborne risks. The Hazard Analysis Critical Control Point System (HACCP) has served to address part of the food safety problem by developing principles used in some food production 
industries. The pairing of a quality HACCP program and a food safety informatics system should lessen the possibility of mass foodborne illnesses.

The Touchblock system has served to demonstrate that technology can efficiently and reliably become a critical proactive tool in the battle to ensure food safety. Federal food regulations within the United States have significantly improved over the last few years, but efficacy of safe food practices across the food industry is woefully lacking. In order to overcome these deficiencies and protect the general public, especially the immune compromised, the young and elderly, the need for bringing about a call to action in food safety through the utilization of advanced technological systems needs to be elevated to the level of a public health imperative, especially at the local levels where foods are produced and consumed.

\section{Conflicts of interest}

The authors have no conflicts of interest to report at this time.

\section{Correspondence}

Cynthia A. Tucker, Ph.D., MBA., RD, LDN

Cynthia.Tucker@Morgan.Edu

Morgan State University

School of Community Health and Policy

Nutritional Science Program

Jenkins Building, Room 329

1700 E. Cold Spring Lane

Baltimore, Maryland 21251

443.885.3561 (Office)

410.235.1737 (Fax)

\section{References}

[1] American Academy of Pediatrics. Children and foodborne illness. Pew Health Group \& Center for Foodborne Illness Research \& Prevention. http://www.makeourfoodsafe.org/tools/Children-and-FBI-Factsheet-11-09.pdf. Accessed 2010, June 3.

[2] Babcock, D. What is it like to live with end stage renal disease (ESRD)? http://foodsafetynews.com/2010/03/living-with-esrd/ 2010, March 15.

[3] Centers for Disease Control and Prevention. 2011. Vital Signs: Incidence and trends of Infection with pathogens transmitted commonly through food - Foodborne diseases Active surveillance network 10 U.S. sites, 1996-2010. MMWR, 1-6.

[4] European Centre for Disease Prevention and Control. Shiga toxin-producing E. coli Update on outbreak in Germany and cluster in France. http://www.foodsafety news.com/tool-in-european-outbreak-continues-to-rise/. Accessed 2011, June 29. 
[5] Food and Drug Administration. Compliance program guidance manual - Part 1, 2009. Retrieved from http://www.fda.gov/downloads/Food/GuideComplianceRegulatory Information/ComplianceEnforcement/UCM193756.pdf. Accessed 2011, June 14.

[6] Food and Drug Administration, US Department of Agriculture, Food Safety and Inspection Service. 2009. Prevention of Salmonella Enteritidis in shell eggs during production, storage and transportation. Final rule. Fed Register, 74:3302-3310.

[7] Gavazzi, G. (2002). Ageing and infection. Lancet Infect Dis, 2, 659-666.

[8] Gerber, C P., Rose, J B., Haas, C N. 1996. Sensitive populations: Who is at the greatest risk? Int J Food Microbiol, 30, 112-123.

[9] Institute for Homeland Security Solutions. 2009. Research Brief: Informatics tools for food safety and defense.North Carolina: Greia, N., Nogueirs, M. Kendall, P., Medeiros, L C., Hiller, V., Gand, C., Dimascola, S. (2003). Food handling behaviors of special importance for pregnant women, infants and young children, the elderly, and immune-compromise people. J Am Diet Assoc, 103, 1646-1649.

[10] Scallan, E., Griffin, P M., Angulo, F J., Tauxe, V., Hoekstra, R M. 2011. Foodborne illness acquired in the United States - unspecified agents. Emerg Infect Dis, 17, 16-22.

[11] Scharff, R L. 2010. Health related cost from foodborne illness in the United States. Washington, DC: Produce Safety Project at Georgetown University; The Pew Charitable.

[12] Schroeder, M S., Kendall, P. Elderly at higher risk of foodborne illness. http://www.ext.colostate.edu/safefood/newsltr/v8n3s05.html. Accessed 2011, June10.

[13] Slotwiner-Nie, P K. 2001. Infectious diarrhea in the elderly. Gastroenteral Clin North Am, 30,625-635.

[14] Sobel, J., Griffith, P M., Slutsker, L., Swerdlow, D L., Tauxe, R V. (2002). Investigation of multi-state foodborne disease outbreaks. Public Health Reports, 117 (1). 8-19.

[15] Stamey, J. 2006. Protecting residents from foodborne illnesses: A dietary perspective on infection control. Long-term Living http://www.ltlmagizine.com/ME2?Segments/Publications. Accessed 2010, June 1.

[16] US Department of Health and Human Services, Center for Disease Control and Prevention. 2008. Prevention, investigation of outbreak of infections caused by Salmonella Saintpaul. www.cdc.gov/salmonells/saintpaul/jalapeno/2008. Accessed 2010, June 13.

[17] US Department of Health and Human Services, Center for Disease Control and Prevention (2006). Update on multi-state outbreak of E. Coli 0157:h7 Infections from fresh spinach www.cdc.gov/foodborne.edolispinach/100606.htm. Accessed 2010, June 13.

[18] Greis, N.P., Nlogueira, M., McDonald, P., Wilfert, R. (2011, May). The NCFODSAFET project: NCFEDA North Carolina Foodborne Events Data Integration and Analysis Tool: A New Informatics Tool for Food Safety in North Carolina. Institute for Homeland Security Solutions. North Carolina Center for Public Health Preparedness 